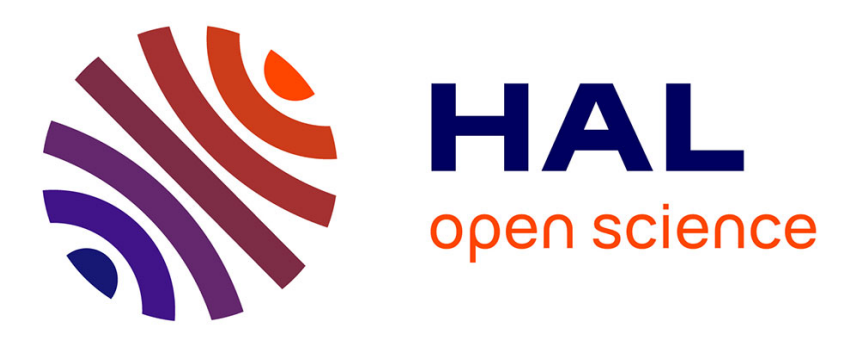

\title{
Light induced flows opposing drainage in foams and thin-films using photosurfactants
}

Eloise Chevallier, Arnaud Saint-Jalmes, Isabelle Cantat, François Lequeux, Cécile Monteux

\section{- To cite this version:}

Eloise Chevallier, Arnaud Saint-Jalmes, Isabelle Cantat, François Lequeux, Cécile Monteux. Light induced flows opposing drainage in foams and thin-films using photosurfactants. Soft Matter, 2013, 9 (29), pp.7054. 10.1039/C3SM50258A . hal-00861319

\section{HAL Id: hal-00861319 https://hal.science/hal-00861319}

Submitted on 24 Sep 2014

HAL is a multi-disciplinary open access archive for the deposit and dissemination of scientific research documents, whether they are published or not. The documents may come from teaching and research institutions in France or abroad, or from public or private research centers.
L'archive ouverte pluridisciplinaire HAL, est destinée au dépôt et à la diffusion de documents scientifiques de niveau recherche, publiés ou non, émanant des établissements d'enseignement et de recherche français ou étrangers, des laboratoires publics ou privés. 


\title{
Soft Matter
}

Cite this: Soft Matter, 2013, 9, 7054

\section{Light induced flows opposing drainage in foams and thin-films using photosurfactants $†$}

\author{
Eloise Chevallier, ${ }^{\mathrm{a}}$ Arnaud Saint-Jalmes, ${ }^{\mathrm{b}}$ Isabelle Cantat, ${ }^{\mathrm{b}}$ François Lequeux ${ }^{\mathrm{a}}$ \\ and Cécile Monteux*a
}

\begin{abstract}
We study the influence of UV light on the drainage flows of foams and thin-liquid films stabilized by photoswitchable azobenzene surfactants, whose shape and hydrophobicity can be modified using UV illumination. This model system, the dynamics of which was well characterized in a previous study, enables us to trigger a controlled variation of the surface excess and surface tension. In both geometries we observe light-induced flows which are able to suppress the drainage flow induced by gravity. However, we show that the physical origin of the flows is different in both geometries. At the scale of a few films in the so-called 'two-bubble' experiment the comparisons of the physical length scales, i.e. the radius of the meniscus and the film thickness, to the chemical "reservoir length" $(I / \mathrm{c})$ show that the flux of the surfactant at the interface in the presence of UV light is different in the films and in the meniscus, inducing a Marangoni flow from the meniscus to the film, which is stronger than gravity and capillary suction. The velocity of this flow can be tuned by the light intensity and the surfactant concentration. In the real foams, however, we show that the above mechanism is not relevant because the radii of curvature of the Plateau borders are orders of magnitude lower than in the two-bubble experiment, thus the capillary suction prevents such transfer between the films and the Plateau borders. Instead, the decrease of the drainage velocity is shown to be due to a gradient of the surface tension in the illuminated zone hence to a local variation of the capillary pressure. This study underlines the importance of characterizing the radius of the Plateau borders for the understanding of foams, as this key parameter sets the order of magnitude of capillary pressure, film thickness and amount of available surfactant. We also show that this photosurfactant is a new toolbox for the understanding of foam stability.
\end{abstract}

Received 24th January 2013

Accepted 26th March 2013

DOI: $10.1039 / c 3 s m 50258 a$

www.rsc.org/softmatter stability and rheology. In fact, both physical parameters (like $\phi_{1}$ and $D$ ) and chemical formulation control the properties of a foam. For instance, the dynamics of foam aging, by drainage and coarsening, can be widely tuned either by changing $D$ or the chemical components. ${ }^{3}$ In addition, the response to shear or the slipping of foams on a substrate combines physical and chemical contributions; ${ }^{4-6}$ the rheology at the interfaces is therefore coupled to the bulk foam rheology. In the process of investigating the effects of the surfactant formulation, it has been observed that some liquid can flow from PBs to films, or within the foam, as a consequence of bubble rearrangements, ${ }^{4,6-9}$ non-uniform shear, ${ }^{10}$ slipping on solid walls, ${ }^{4,11,12}$ or in association with drainage flow in the PBs. ${ }^{13-15}$ Qualitatively, such bubble rearrangements or liquid flow in PBs induce surfactant convection in bulk and at interfaces, eventually resulting in surface tension gradients. Local Marangoni flows then occur in response to these surface tension gradients. These local flows, by changing the sizes of the films and PBs, might then react and have an impact on the dynamical forcing at their origins. Thus, these are strongly coupled problems, which need to be investigated if one wants a full understanding of foams in dynamical conditions. However, the surface tension, surfactant
${ }^{a}$ Soft Matter Science and Engineering UMR7615 UPMC/CNRS/ESPCI ParisTech, 10 rue Vauquelin, 75231 Paris, France. E-mail: francois.lequeux@espci.fr

${ }^{b}$ Institut de Physique de Rennes, France

† Electronic supplementary information (ESI) available. See DOI: $10.1039 / \mathrm{c} 3 \mathrm{sm} 50258 \mathrm{a}$ 
concentration and film thickness cannot be determined experimentally in situ in a foam.

As an alternative, some experiments were performed on elementary foam structures, consisting of only one or a few bubbles/films. ${ }^{7,9,11-13}$ However, one can always wonder if these results remain valid within a foam, and whatever the typical sizes of the bubbles and Plateau borders.

In this study, to overcome some of these drawbacks, our strategy is twofold. First, it consists of using a photosurfactant, whose shape and hydrophobicity can be tuned by UV light stimulation. As we have shown in a previous study ${ }^{16}$ (summarized in Section B) the two isomers of the azobenzene photosurfactant have very different adsorption dynamics and equilibrium surface excess. Here we use this surfactant as a tool to tune the surfactant properties in situ inside foams and in thin-liquid films by switching on and off the light stimulation and to monitor the induced flows. With this well-characterized model, we also have a way to properly trigger photoinduced spatial and time variation of the surface excess and then to generate Marangoni flows. Secondly, we choose to investigate two different geometries: a model setup based on three thinliquid films of a few $\mathrm{cm}$ sizes and a 3D foam, where the bubbles have a sub-millimeter radius.

In both geometries, we find clear evidence of flow triggered by the UV light stimulation: in all cases, these light induced effects are stronger than gravity and capillarity. We suggest theoretical models to explain these flows. We also discuss the link between the two set-ups: we show that in the two-bubble experiment the relevant physical dimensions (typically of the order of $\mathrm{mm}$ to $\mathrm{cm}$ ) are orders of magnitude different from the ones within the foam (scaled down to microns), leading to two different interpretations of the light-induced hydrodynamic effects.

Finally, we confirm that the use of these photoactive surfactants provides a new toolbox for the understanding of the link between surfactant properties and foam stability.

\section{B Photoswitchable surfactants}

Photoswitchable surfactants have emerged in the last 20 years as a way to control interfacial dynamics. ${ }^{17,18}$ The photoswitchable surfactant we used is called AzoTAB, which contains an azobenzene moiety on the hydrophobic tail and can switch from the cis to the trans-isomer under UV or blue stimulation (Fig. 1).

Details on the synthesis of the photosurfactant and measurement of the cis : trans ratio as a function of wavelength are given in our previous study. ${ }^{16}$ Briefly, under blue light $(\lambda=$ $436 \mathrm{~nm}$ ) the solution reaches a photostationary state containing a predominant fraction of trans-isomers ( $\left.\phi_{\text {blue }}=66 \%\right)$. Under UV light $(\lambda=365 \mathrm{~nm})$, the surfactant solution contains $\phi_{\mathrm{UV}}=$ $5 \%$ of the trans-isomers. The typical time for switching the bulk composition from one state to the other scales as $\tau_{\text {photo }}=1 / I=$ $1 /[a(I)+b(I)]$, where $I$ is the light intensity, and $a(I)$ and $b(I)$ are the trans-to-cis and cis-to-trans photoconversion constants in $\mathrm{s}^{-1}$, respectively. From spectroscopic measurements, ${ }^{16}$ we found that $a(I) \sim 5 \times 10^{-2} I$ and $b(I) \sim 2 \times 10^{-3} I$. As $a(I) \gg b(I)$, we will neglect $b(I)$ in the following.

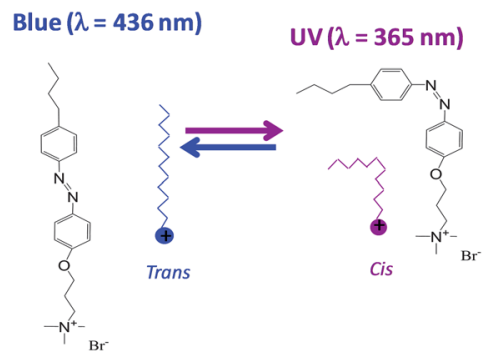

Fig. 1 Trans and cis forms of the azobenzene based photosurfactants.

In ref. 16 we also investigated the short time adsorption dynamics of cis-trans mixtures using dynamical surface tension measurements. We determined the desorption and adsorption constants of the trans-isomers: $k_{\text {des }}^{\text {trans }} \sim 5 \times 10^{-7} \mathrm{~mol} \mathrm{~m}^{-2} \mathrm{~s}^{-1}$ and $k_{\text {ads }}^{\text {trans }} \sim 5 \times 10^{-7} \mathrm{~m} \mathrm{~s}^{-1}$ as well as the ones of the cis-isomers $k_{\text {des }}^{\text {cis }} \sim 10^{-4} \mathrm{~mol} \mathrm{~m}^{-2} \mathrm{~s}^{-1}$ and $k_{\text {ads }}^{\text {cis }} \sim 10^{-6} \mathrm{~mol} \mathrm{~m}^{-2} \mathrm{~s}^{-1}$. . These constants can be used to deduce the typical adsorption/ desorption times of both isomers. We showed that, in the dark, at equilibrium, the liquid-air interface is mostly covered with trans-isomers because the desorption of the cis-isomers is faster than that of the trans by 2 orders of magnitude. When UV or blue light is applied on the trans-rich interface, the adsorbed trans are photoconverted into cis-isomers with a typical time $\tau_{\text {photo }}$. The freshly converted cis-isomers desorb with a typical time $\tau_{\text {des }}^{\text {cis }}$ such that $\tau_{\text {des }}^{\text {cis }} \ll \tau_{\text {photo }} \ll \tau_{\text {ads }}^{\text {trans }}$, where $\tau_{\text {ads }}^{\text {trans }}$ is the typical time for the trans adsorption. It follows that the desorption flux of the cis-isomers is limited by the rate of photoconversion and that the trans isomers do not adsorb fast enough to replenish the interface. As a result, the surface excess decreases with the light intensity and the surface tension increases with the light intensity. In this study, solutions of azosurfactant, from $2 \mathrm{mM}$ to $46 \mathrm{mM}$ in deionized water, were exposed for 30 minutes under blue light before the experiments using a Prizmatix device equipped with a photodiode $(436 \mathrm{~nm})$, $I=0.4 \mathrm{~mW} \mathrm{~cm}^{-2}$, injection system in fiber optics, and output collimation optics. To investigate the effect of UV light and trans to cis photoconversion on the thin-films and foams, UV light was shone at a maximal power of $I=1 \mathrm{~mW} \mathrm{~cm}{ }^{-2}$ using the same Prizmatix device equipped with another photodiode (365 nm). The same fiber output was used to irradiate foams and thinfilms at a distance of $c a .1 \mathrm{~cm}$. We verified that for these intensities there is no effect of a rise of temperature: measurements using a thermal camera show that the temperature stays constant in the foam.

\section{Photoinduced flows at the scale of several thin-films}

We investigate the influence of UV light on the drainage in a vertical thin-liquid film connected between two open bubbles

$\ddagger$ The times for the dynamic surface tension measurements given in ref. 16 were erroneously renormalized by a factor $Z^{2}=100$ as suggested in ref. 26 . We found out that the correct value of this apparatus constant is in fact equal to 1 . The adsorption/desorption constants given in ref. 16 are thus surestimated by a factor 100. The values given in Section B of this article are the correct ones. 
(Fig. 2a). Before stimulation, the thin-film drains under the action of gravity and becomes thicker at the bottom, as shown by the Newton fringes in Fig. $2 \mathrm{~b}$, for $t=0 \mathrm{~s}$. When UV light is shone on the sample at $t=0$, the film stops thinning and an upward flow tends to thicken the film. The brown fringe, characteristic of a thickness $h=140 \mathrm{~nm}$, moves upward (Fig. 2b). This first experiment is clear evidence that UV light and photosurfactants can be used to achieve a remote control of the drainage flows in thin-liquid films.

To investigate the light-induced flows without the effect of gravity, we rotated the set-up shown in Fig. 2 until the thin-film is horizontal. As shown in Fig. 3, the UV stimulation results in a radial flow directed toward its center. A fresh film of uniform thickness $H$ of the order of one $\mu \mathrm{m}$ is drawn from the Plateau borders and invades the central film of initial thickness $h_{0}$ of the order of 300 to $750 \mathrm{~nm}$.

The area $A(t)$ of the thin-film initially present decreases with time at a constant rate (Fig. $3 \mathrm{c}$ ), from its initial value $A_{0}$. However, the volume of the central film, $h_{0} \times A(t)$, is constant during the experiment, meaning that there is no transfer of material between the initial thin-film and the fresh film of thickness $H$ that invades the thin film.

This situation is similar to the problem described theoretically by Frankel where a soap film of thickness $H$ is mechanically drawn from a bath of liquid at a velocity $U$ using a metal frame. ${ }^{19}$ In the steady state, the thickness $H$ results from the balance between capillary forces and viscous dissipation in the film and is given theoretically by $H=2.68 r_{\mathrm{m}} \mathrm{Ca}^{2 / 3}$, with $r_{\mathrm{m}}$ the radius of curvature of the meniscus, $\mathrm{Ca}=\eta U / \gamma$ the capillary number, $\gamma$ the surface tension, and $\eta$ the viscosity. In Frankel's situation the curvature of the meniscus is set by $\frac{l_{\mathrm{c}}}{\sqrt{2}}$ with $l_{\mathrm{c}}=\sqrt{\frac{\rho g}{\gamma}}$ the capillary length, $\rho$ the liquid density and $g$ the

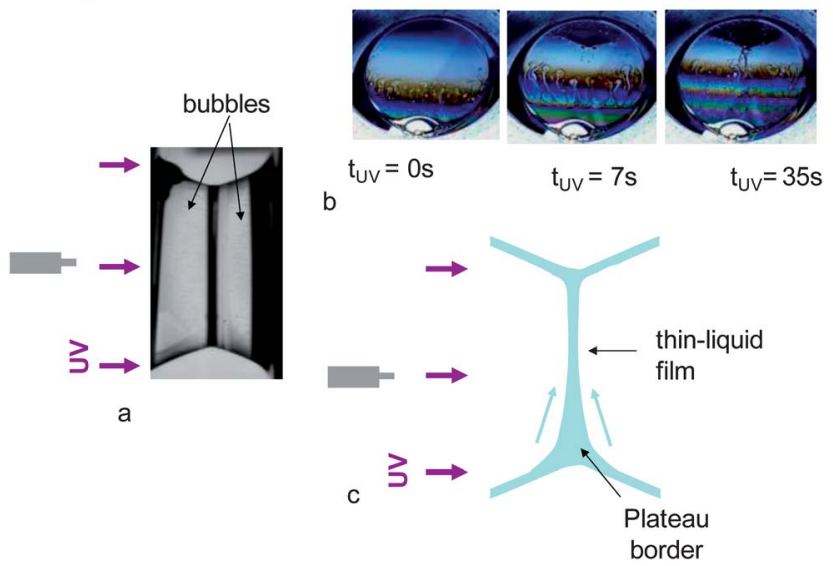

Fig. 2 (a) Set-up allowing the formation of a vertical thin-film between two lateral films and illumination with a UV light; ( $b$ and c) - effect of UV light on the drainage. In (b), the series of photos show the central thin-film observed with white-light in reflection. In the dark, gravity induces a downward flow which tends to thicken the lower part of the film. When the UV light is switched on, an upward flow is opposing the drainage flow. The brown fringe $(h=140 \mathrm{~nm}$ ) moves upward. See video in ESIt.
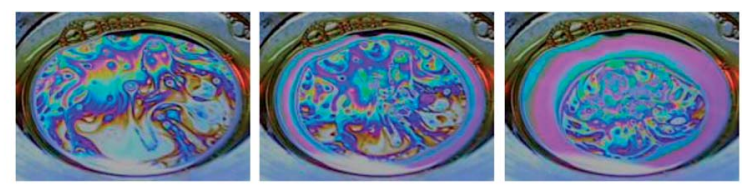

$3 a$

$3 \mathrm{~b}$
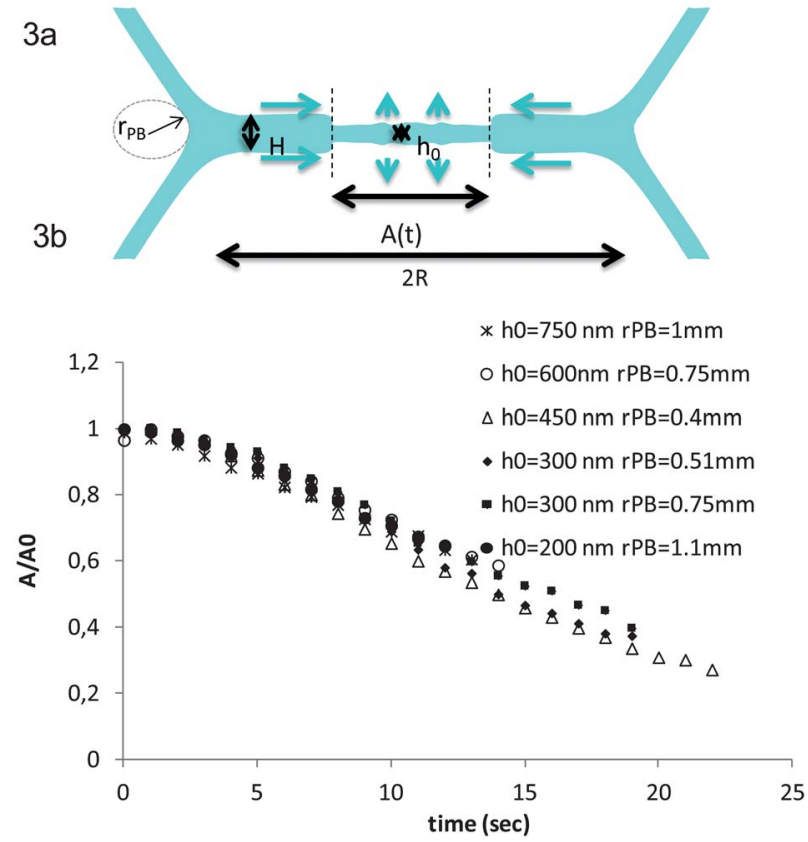

Fig. 3 (a) A horizontal film illuminated with UV light at time $t=0,6$, and 12 seconds after UV light is switched on. A fresh film of thickness $H$ invades the thin film of initial thickness $h_{\text {moy. }}$ (b) Schematic side view of the flow presented in the photos of (a). (c) Area of the central film as a function of time for various conditions such as initial thickness $h_{\text {moy }}$ and radius of curvature $r_{\mathrm{PB}}$.

gravity constant, whereas in our case the relevant length scale is given by the Plateau borders, whose curvature, $r_{\mathrm{PB}}$, can be controlled by injecting a liquid using a syringe into the menisci.

Unlike in Frankel's work where a direct mechanical traction is needed to pull the film, applying UV light on the interface enables us to control remotely the advance of the fresh film. Moreover varying the light intensity or the surfactant concentration enables us to tune the velocity $U$ and hence to explore different capillary numbers between $10^{-5}$ and $10^{-4} \cdot r_{\mathrm{PB}}$ is measured by image analysis of the profile of the Plateau border, $H$ is measured by interferometry and the velocity $U$ is obtained by measuring $\mathrm{d} R / \mathrm{d} t$, the rate at which the radius of the central film decreases. We find that the ratio $H / r_{\mathrm{PB}}$ of the thickness $H$ and the radius of the Plateau borders scale as $\mathrm{Ca}^{2 / 3}$ as predicted by Frankel (Fig. 4). Our results fall slightly below Frankel's prediction, as also observed for several solutions in Frankel's geometry. ${ }^{20}$ This likely indicates that the interfaces of the photoswitchable surfactants may exhibit some dilations whereas Frankel assumed rigid interfaces.

Let us discuss the driving force for this light-induced Frankel flow. It was shown recently that pulling a film at a velocity $U$ requires a surface tension difference between the menisci and the film given by ${ }^{\mathbf{2 1}}$

$$
\delta \gamma=5 \gamma \mathrm{Ca}^{2 / 3}
$$




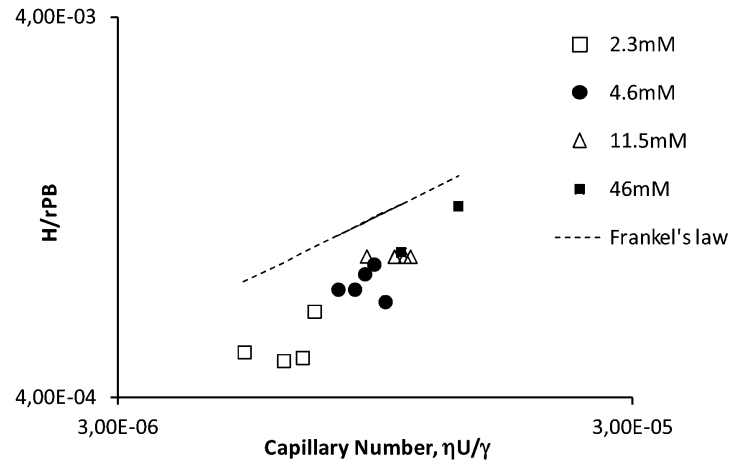

Fig. 4 Ratio of thickness $H$ of the fresh film drawn from the meniscus to the radius of the Plateau borders $r_{\mathrm{PB}}$ as a function of the capillary number, $\mathrm{Ca}=\eta U / \gamma_{\text {, }}$ where $U$ is the advancing velocity of the fresh film. The dotted line represents Frankel's theoretical law.

From our measurements of $U$ we estimate that $\delta \gamma$ is of the order of $10^{-3}$ to $10^{-2} \mathrm{mN} \mathrm{m}^{-1}$. Using a standard Langmuir isotherm,

$$
\gamma=\gamma_{0}+2 R T \Gamma_{\infty} \ln \left(1-\frac{\Gamma}{\Gamma_{\infty}}\right)
$$

where $\Gamma_{\infty}$ is the surface excess for a saturated interface, we estimate that this corresponds to a small difference of the surface excess, $\delta \Gamma$ of less than $1 \%$ between the film and the meniscus.

We have shown previously ${ }^{\mathbf{1 6}}$ that the adsorption of the cis- $^{-}$ isomers is negligible. Thus we will limit ourselves to an approximate balance that includes only the trans-isomer. Let us first write the balance equation for the surface excess of transisomers $\Gamma^{\text {trans }}$ on the interfaces of the thin-film as follows

$$
\begin{aligned}
\frac{\mathrm{d} \Gamma^{\mathrm{trans}}}{\mathrm{d} t}= & k_{\text {ads }}^{\text {trans }} c_{\text {trans }}\left(1-\frac{\Gamma^{\mathrm{trans}}}{\Gamma_{\infty}^{\mathrm{trans}}}\right)-k_{\mathrm{des}}^{\mathrm{trans}}\left(\frac{\Gamma^{\mathrm{trans}}}{\Gamma_{\infty}^{\mathrm{trans}}}\right)-a(I) \Gamma^{\mathrm{trans}} \\
& -\left(\frac{\Gamma^{\mathrm{trans}}}{A} \frac{\mathrm{d} A}{\mathrm{~d} t}\right),
\end{aligned}
$$

where $A$ is the area of the central film, $c_{\text {trans }}$ is the trans concentration in the solution, $k_{\mathrm{ads}}^{\text {trans }}, k_{\mathrm{des}}^{\text {trans }}$ and $a(I)$ are defined in Section B. The first two terms of the r.h.s. correspond to the surfactant exchange with the bulk. The third one corresponds to the light-induced flux of desorption of the adsorbed trans, as explained in Section B. The fourth term corresponds to the change of surface coverage originating from surface compression of the trans layer in the central film due to the advance of the Frankel film. The same equation holds for the meniscus. However we will assume that the meniscus area is constant, consistent with Frankel's assumption of rigid interface close to the meniscus. The last term of eqn (3) thus vanishes in the meniscus case.

For the concentrations we used $h_{0}<\Gamma_{\infty}^{\text {trans }} / c_{\text {trans }} \sim 1 \mu \mathrm{m} \ll$ $r_{\mathrm{PB}}$, hence under illumination the equilibrium between the interface and the bulk is different in the meniscus and in the thin-film. As a result, $c_{\text {trans }}$ is slightly lower in the film than in the meniscus which can be viewed as a reservoir. Hence the surface excess is different between the meniscus and the film. Thus the UV illumination generates a Marangoni flow between the film and the meniscus, which induces the thin-film retraction and the formation of the Frankel's film, as we modelize below.

To predict the retraction rate of the central film, $\varepsilon=1 / A(\mathrm{~d} A / \mathrm{d} t)$ in $\mathrm{s}^{-1}$ we have to derive the expression for the concentration and surface excess of the trans-isomers in the film and in the meniscus as a function of time under UV illumination. The total amount of trans-isomer $N^{\text {trans }}$ decreases with the following law, both in the film (denoted with index $f$ ) and in the meniscus (denoted with index $m$ )

$$
N_{\mathrm{f} / \mathrm{m}}^{\mathrm{trans}}=N_{\mathrm{f} / \mathrm{m}} \phi_{\mathrm{UV}}+\left(N_{\mathrm{f} / \mathrm{m}}^{\mathrm{trans}}(0)-N_{\mathrm{f} / \mathrm{m}} \phi_{\mathrm{UV}}\right) \mathrm{e}^{-t / \tau \text { photo }}
$$

with $N_{\mathrm{f} / \mathrm{m}}$ the total amount of surfactant, $\phi_{\mathrm{UV}}=0.05$ and $\tau_{\text {photo }} \sim$ $1 / a(I)$ the photoconversion time as defined in Section B. In the meniscus, the amount of surfactant at the interface is negligible with respect to the bulk. Thus the initial amounts of transisomer and surfactant are simply given by $N_{\mathrm{m}}^{\text {trans }}(0)=c \phi_{\text {blue }} V_{\mathrm{m}}$, with $V_{\mathrm{m}}$ the meniscus volume, $c$ the concentration and $\phi_{\mathrm{blue}}=$ 0.66 and $N_{\mathrm{m}}=c V_{\mathrm{m}}$, respectively. The concentration is $c_{\mathrm{m}}^{\text {trans }}(t)=$ $N_{\mathrm{m}}^{\text {trans }}(t) / V_{\mathrm{m}}$. Then, from eqn (3) we deduce $\Gamma_{\mathrm{m}}^{\text {trans }}$, the surface excess on the meniscus as a function of time under UV illumination.

In the thin-film the interface dominates. The initial amounts of trans-isomer and surfactant are $N_{\mathrm{f}}^{\text {trans }}(0)=c \phi_{\text {blue }} V_{\mathrm{f}}+$ $2 A_{0} \Gamma_{\text {eq }}^{\text {trans }}(c)$ and $N_{\mathrm{f}}(0)=c V_{\mathrm{f}}+2 A_{0} \Gamma_{\text {eq }}^{\text {trans }}(c)$, respectively. The concentration in the film $c_{\mathrm{f}}^{\text {trans }}(t)$ depends on the total amount of trans-isomer $N_{\mathrm{f}}^{\text {trans }}(t)$ but also on the area of the interface through the relationship

$$
c_{\mathrm{f}}^{\text {trans }}(t)=\frac{1}{V_{\mathrm{f}}}\left(N_{\mathrm{f}}^{\text {trans }}(t)-2 \Gamma_{\mathrm{f}}^{\text {trans }}(t) A(t)\right)
$$

Eqn (3)-(5) constitute a set of coupled equations that can be closed using the fact that the surface excess difference between the film and the meniscus remains of the order of $1 \%$ : the area of the film adapts itself in order to maintain at each time the same surface excess everywhere, despite the different surfactant dynamics in the meniscus and in the film. In eqn (3), written for the film, we thus replace $\Gamma_{\mathrm{f}}^{\text {trans }}$ by the expression found for $\Gamma_{\mathrm{m}}^{\text {trans }}$. This differential equation involves the single unknown $A(t)$ and is solved using Matlab. The residual difference of the surface excess, $\Gamma_{\mathrm{m}}^{\text {trans }}-\Gamma_{\mathrm{f}}^{\text {trans }}$, induces a Marangoni stress needed to pull the Frankel's film, but it can be neglected to predict the area evolution.

In Fig. 5 we show that the rate of compression of the central film $\varepsilon=(1 / A)(\mathrm{d} A / \mathrm{d} t)$ measured experimentally increases almost linearly with the light intensity and increases with the concentration. The initial film thickness does not have any significant influence on the dynamics (see Fig. 3). These qualitative behaviours are perfectly reproduced by our model.

The full lines shown in Fig. $5 \mathrm{a}$ and $\mathrm{b}$ are the predictions obtained from the model using $k_{\mathrm{ads}}^{\text {trans }}=10^{-7} \mathrm{~m} \mathrm{~s}^{-1}$ and $k_{\mathrm{des}}^{\text {trans }}=$ $10^{-7} \mathrm{~mol} \mathrm{~m}^{-2} \mathrm{~s}^{-1}$ and $a(I)=0.09 I \mathrm{~s}^{-1}$, which are in very good agreement with the values obtained independently in our previous study. ${ }^{\mathbf{1 6}}$

Using our photoswitchable surfactants, we thus generate a controlled Marangoni flow from the Plateau borders originating from a difference in exchange dynamics of the surfactants 

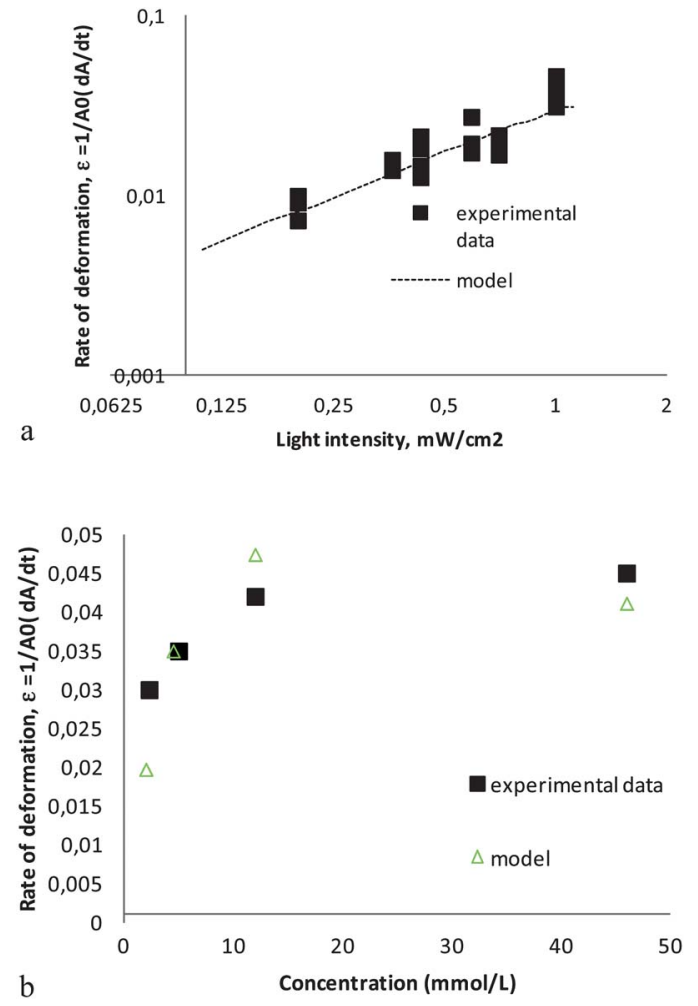

Fig. 5 (a) Rate of retraction, $\varepsilon$, of the central film as a function of light intensity. The squares represent the data points and the line represents the model. (b) Rate of retraction of the fresh film as a function of the surfactant concentration. The squares represent the data points and the triangles represent the model.

between the meniscus and the film under UV light. The velocity is controlled by the light intensity and hence the rate of cis-trans photoconversion at the interface. Below we study light-induced flows at the scale of a macroscopic 3D foam.

\section{Photoinduced flows at the scale of a macroscopic foam}

A $30 \mathrm{~cm}$ high column with a rectangle section of $5 \mathrm{~cm}^{2}$ is filled with an aqueous foam made with the photosurfactant solution at a concentration $1.15 \mathrm{mM}$. The foam is produced by bubbling gas through porous frits of controlled porosity, and at controlled pressure. We illuminate a $1 \mathrm{~cm}$ wide horizontal section of the foam using fiber optics (Prizmatix) while the other part is kept in the dark. We record the time evolution of the liquid fraction at several heights in the foam using conductimetric measurements according to the method described in ref. 22. In the absence of UV light, the liquid fraction $\phi_{1}$ recorded by the electrodes at all heights decreases monotonously with time because of the drainage flow induced by gravity, similarly to standard surfactants (ref. 3). A typical drainage curve $\phi_{1}(t)$ without UV light is shown in Fig. 6a. When UV light is applied locally on a given section of the foam, as described in more detail below, the liquid fraction of the illuminated section stops decreasing or even increases for several hundreds of seconds (Fig. 6c). For the rest of the foam, kept in the dark, we do not detect significant variations in the curve $\phi_{1}(t)$ (not shown).

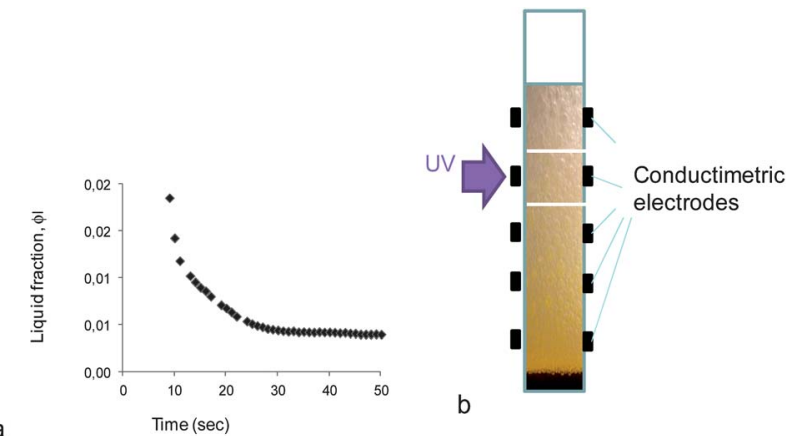

a

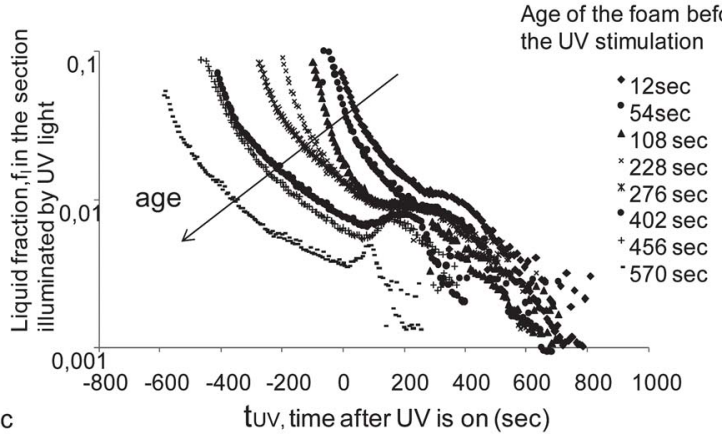

Fig. 6 ( $a$ and b) Set-up used to study the influence of UV light on foam drainage. Conductimetric electrodes enable us to obtain the liquid fraction inside the foam as a function of the height in the foam column. (a) A typical drainage curve obtained in the dark. (c) Time variation of the liquid fraction of the section of the foam illuminated by UV light. The results are given for foams which have been "aged", i.e. left to drain in the dark for times between 12 and 570 seconds. Before UV stimulation the liquid fraction decreases as a function of time because of the drainage flow induced by gravity. When the UV light is shone on the foam column, the liquid fraction of the illuminated section of the foam remains constant or even swells with liquid.

Varying the age of the foam at which the UV light is turned on enables us to explore the influence of the initial liquid fraction of the foam. For young and humid foams whose initial liquid fraction is above $1 \%$ when shone in UV the liquid fraction remains constant for typically 200 seconds after switching on the UV light. After $200 \mathrm{~s}$, the foam starts breaking similarly to what we reported in a previous article. ${ }^{23}$ For dryer foams $-\phi_{1}<$ $1 \%$ aged for more than $400 \mathrm{~s}$, the UV stimulation leads to an increase of the liquid fraction for 100 seconds and then the foam starts breaking.

As for the thin-film experiment, we observe a flow which can actually be stronger than gravity. Concerning the mechanism at the bubble scale, allowing the foam to remain locally wet, one can wonder if the liquid transfer from PBs towards films, described in the previous section, can also be relevant here. Indeed, redistribution of the liquid in the films, from the PB, could explain the slowing down of the global drainage (even with swelling, the film thickness would still remain small compared to $r_{\mathrm{pb}}$ ) Thus one first has to figure out what would be the film thickness in the foams. Following Section C, we have derived the relationship $H \sim r_{\mathrm{PB}} \mathrm{Ca}^{2 / 3}$. In the experimental foam, $r_{\mathrm{PB}}$ depends on $\phi_{1}$ according to $r_{\mathrm{PB}} \approx 2.5 \phi_{1}^{1 / 2} L_{\mathrm{PB}}$ eqn (5), where $L_{\mathrm{PB}}$ is the length of the Plateau borders. ${ }^{1,2,24}$ For values of $\phi_{1}=$ 0.01 and $L_{\mathrm{PB}}=200 \mu \mathrm{m}$, typical for our dry foams, we estimate 
that $r_{\mathrm{PB}}$ is of the order of $6 \mu \mathrm{m}$, almost 2 orders of magnitude smaller than in the two bubble-experiment. Extrapolating the experimental Frankel's law obtained in Fig. 4 to such low values of $r_{\mathrm{PB}}$ would thus lead to the very small value of $H$, typically $3 \mathrm{~nm}$. This has to be compared to the thickness existing in such foams, before the illumination. Because $r_{\mathrm{pb}}$ is a few microns, we obtain capillary pressures of hundreds of Pascals, implying high disjoining pressures between the charged azoTAB monolayers of the films and thickness of the order of 10 to $50 \mathrm{~nm}$ (as estimated from typical disjoining pressure isotherms measured for standard cationic surfactants ${ }^{25}$ ).

Hence the capillary suction, which is much higher in the dry foams than in the 2-bubble experiment, induces film thickness higher than what the Frankel flow between the Plateau borders and the films would induce. We can thus discard the previous PB-film mechanism for the behavior in the foams. In fact, a more advanced version of this model should include disjoining pressure effects, in order to introduce a cut-off thickness, when the $\mathrm{PB}$ radius decreases and when the capillary pressure becomes too high.

Even for the most humid foams, for which $r_{\mathrm{PB}}$ is higher, this mechanism remains not relevant. In fact, we can probably explain that drainage is poorly affected by UV light for the wet foams: we suggest that the portion of the illuminated foam decreases for higher initial liquid fraction. In these foams, light is always multiply scattered, and its propagation is characterized by a light mean free path, $1^{*}$. The amount of light transmitted through a foam slab is then proportional to this length. It turns out that $1^{*}$ is inversely proportional to the liquid fraction. ${ }^{22}$ Thus, light is less penetrating, and more backscattered, as the liquid fraction increases. This explains why the inverse drainage gradually vanishes for the wettest foams, as there is a smaller fraction of the foam which is illuminated.

Once we have discarded the interpretation valid for the film experiments, we instead suggest that considering only the network of PBs, the modification of the rate of drainage in the illuminated section of the foam is due to a gradient of capillary pressure caused by the gradient of surface tension between the stimulated part and the rest of the foam. Locally, in the PBs, the surface tension increases, meaning that the capillary pressure (bubble gas pressure - liquid pressure in the $\mathrm{PB}$ ) increases. Therefore, the liquid pressure decreases in the stimulated region. Such a vertical gradient should induce a capillary flow towards the region where the liquid pressure is the lower. Note that this effect is superposed to the overall drainage flow. According to Darcy's law, the draining velocity, $u(z)$, of water inside a foam results from hydrostatic and capillary pressure gradients and writes

$$
u(z)=\frac{K\left(\phi_{1}\right)}{\eta}\left(\rho g-\nabla P_{\mathrm{c}}\right)
$$

where the vertical gradient of capillary pressure is given by the Laplace equation

$$
\nabla P_{\mathrm{c}}=-\nabla\left(\frac{\gamma}{r_{\mathrm{PB}}}\right)
$$

and $K\left(\phi_{1}\right)$ is the permeability of the foam. Besides, according to mass conservation, at a given height of the foam, $\phi_{1}(z)$ varies with time according to

$$
\frac{\partial \phi_{1}(z)}{\partial t}=\frac{\partial u(z)}{\partial z}
$$

In our case the draining velocity $u(z)$ is lower in the illuminated section than in the rest of the foam because the surface tension is higher, driving the accumulation of liquid in this zone. We write the variation of drainage velocity between the sections in the dark and the stimulated one,

$$
\delta\left(\frac{\partial \phi_{1}}{\partial t}\right)=\frac{K\left(\phi_{1}\right)}{\eta r_{\mathrm{PB}}} \frac{\partial^{2} \delta \gamma}{\partial z^{2}} .
$$

Knowing that $K\left(\phi_{1}\right) \approx C_{\mathrm{v}} L_{\mathrm{PB}}{ }^{2} \phi_{1}{ }^{2}$ in eqn (6),, ${ }^{\mathbf{1 , 2 , 2 1}}$ where $C_{\mathrm{v}} \approx$ $10^{-3}$ is a coefficient linked to the triangular geometry of the foam PB, and using eqn (5), we obtain

$$
\delta\left(\frac{\partial \phi_{1}}{\partial t}\right) \approx \frac{0.4 C_{\mathrm{v}} L_{\mathrm{PB}} \phi_{1}^{3 / 2} \delta \gamma}{\eta \Delta_{\mathrm{UV}}{ }^{2}}
$$

with $\Delta_{\mathrm{UV}} \sim 10^{-2} \mathrm{~m}$, the size of the zone which is illuminated with the UV light. Taking $\delta \gamma=10 \mathrm{mN} \mathrm{m}^{-1}, L_{\mathrm{PB}}=200 \mu \mathrm{m}$ and $\phi_{1}=0.4$ to $4 \%$, we find $\delta\left(\frac{\partial \phi_{1}}{\partial t}\right)=2 \times 10^{-6}$ to $6 \times 10^{-5} \mathrm{~s}^{-1}$. From Fig. $6 \mathrm{c}$, we obtain experimentally $\delta\left(\frac{\partial \phi_{1}}{\partial t}\right)=\left(\frac{\partial \phi_{1}}{\partial t}\right)_{t_{\mathrm{UV}=30 \mathrm{~s}}}-\left(\frac{\partial \phi_{1}}{\partial t}\right)_{t_{\mathrm{UV}=-30 \mathrm{~s}}}$ by extracting the slopes of the $\phi_{1}(t)$ curves $30 \mathrm{~s}$ before and $30 \mathrm{~s}$ after the UV is switched on. For $\phi_{1}=0.4$ and $4 \%$ (foam ages 54 and 570 seconds respectively) we find $\delta\left(\frac{\partial \phi_{1}}{\partial t}\right)=4 \times 10^{-5}$ and $9 \times 10^{-5} \mathrm{~s}^{-1}$, which is in good agreement with the values given by the model. These results confirm that light induced variation of the surface tension at the scale of the PBs can induce variations of the drainage velocity inside the foams.

Finally, we focused here on the slowing down of the drainage under stimulation, which can be considered as a source of increased "stability" for a foam (as the dryer the foam, the less stable it is); however, as seen in Fig. 6, this stabilizing effect is rapidly replaced by a foam collapse, described in ref. 21, which also deserves to be studied. Understanding the origins of this collapse brings us back to the scale of the thin films, and to discuss the balance between hydrodynamical instability and vanishing disjoining pressure (vanishing repulsive interaction as the interfaces are less covered). This will be the object of a future work.

\section{F Conclusion}

We have studied photoinduced flows in thin-films and in foams using a photosurfactant. In both geometries we find that it is possible to slow down the drainage using light induced gradients of surface tensions. Comparing the two experiments - film and foam - and the details of each mechanism, it turns out that the $\mathrm{PB}$ radius is several orders of magnitude higher in the twobubble experiment than in the real foams. It follows that the capillary suction, dynamics of surfactant exchange, the 
equilibrium and dynamical thickness are different in both experiments. Hence the flows observed at both scales have a different physical origin. In the two-bubble experiment, the comparisons of the physical scales, $r_{\mathrm{pb}}$ and $h$, to the chemical "reservoir length" $(\Gamma / c)$ show that the flux of the surfactant at the interface in the presence of UV light is different in the films and in the $\mathrm{PB}$, inducing a Marangoni flow from the $\mathrm{PB}$ to the film, which is stronger than gravity and capillary suction. In the foams, however, a gradient of variation of the surface tension in the illuminated zone induces a local variation of the capillary pressure, hence a vertical modulation of the drainage velocity.

We believe that the use of these photoactive surfactants provides a new toolbox for controlling emulsion and foam drainage as well as for the understanding of the link between the surfactant properties and foam stability. In fact, understanding the origin of the foam collapse in this system will be the object of future work.

\section{Acknowledgements}

The present work was supported by Université Pierre et Marie Curie (PhD fellowship) and CNRS. CM thanks V. Bergeron for early discussions on the photosurfactants, C. Tribet for sharing with us his expertise in azobenzen amphiphiles, as well as P. Rouschmeyer for his preliminary experiments on the Marangoni flows.

\section{Notes and references}

1 The Physics of Foams, S. Hutler and D. Weaire, Clarendon Press, Oxford, 1999.

2 I. Cantat et al., Les mousses, Structure et dynamique, Collection Echelles, Belin, 2010.

3 A. Saint-Jalmes, Soft Matter, 2006, 2, 836.

4 N. D. Denkov, S. Tcholakova, K. Golemanov, V. Subramanian and A. Lips, Colloids Surf., A, 2006, 282, 329.

5 S. Marze, D. Langevin and A. Saint-Jalmes, J. Rheol., 2008, 52(5), 1091.
6 N. D. Denkov, S. Tcholakova, K. Golemanov and A. Lips, Phys. Rev. Lett., 2009, 103, 118302.

7 A. L. Biance, S. Cohen-Addad and R. Hoehler, Soft Matter, 2009, 5, 4672-4679.

8 A. Colin and V. Carrier, Langmuir, 2003, 19, 4535-4538.

9 A. L. Biance, A. Delbos and O. Pitois, Phys. Rev. Lett., 2011, 106, 068301.

10 S. Marze, A. Saint-Jalmes and D. Langevin, Colloids Surf., A, 2005, 263, 121.

11 J. Emile, S. Hardy, G. Ropars, A. Saint-Jalmes and R. Delannay, Colloids Surf., A, 2007, 304, 72-76.

12 I. Cantat and B. Dollet, Soft Matter, 2012, 8, 7790-7796.

13 O. Pitois, C. Fritz and M. Vignes-Adler, Colloids Surf., A, 2005, 261, 109.

14 O. Pitois, C. Fritz and M. Vignes-Adler, J. Colloid Interface Sci., 2005, 282, 458.

15 O. Pitois, N. Louvet and F. Rouyer, Eur. Phys. J. E, 2009, 30, 27-35.

16 E. Chevallier, A. Mamane, H. A. Stone, C. Tribet, F. Lequeux and C. Monteux, Soft Matter, 2011, 7, 7866.

17 J. Y. Shin and N. L. Abbott, Langmuir, 1999, 15, 4404.

18 T. Shang, L. A. Smith and T. A. Hatton, Langmuir, 2003, 19, 10764.

19 K. J. Mysels, K. Shinoda and S. Frankel, Soap films: studies of their thinning, New York, Pergamon Press, 1959.

20 J. Lal and J. M. Di Meglio, J. Colloid Interface Sci., 1994, 164, 506-509.

21 I. Cantat, Phys. Fluids, 2013, 25, 031303.

22 K. Feitosa, S. Marze, A. Saint-Jalmes and D. J. Durian, J. Phys.: Condens. Matter, 2005, 17, 6301.

23 E. Chevallier, C. Monteux, F. Lequeux and C. Tribet, Langmuir, 2012, 28, 2308.

24 S. A. Koehler, S. Hilgenfeldt and H. A. Stone, Langmuir, 2000, 16, 6327.

25 V. Bergeron, Langmuir, 1997, 13, 3474.

26 N. C. Christov, K. D. Danov, P. A. Kralchevsky, K. P. Ananthapadmanabhan and A. Lips, Langmuir, 2006, 22, 7528-7542. 